\title{
Transverse Mode Control and Switching in Gas Laser Arrays
}

\author{
Yongfang Zhang and William B. Bridges, Fellow, IEEE
}

\begin{abstract}
Theoretical and experimental investigations of multiple transverse mode laser oscillation involving spatially varying gain and loss are carried out. The effect of gain and loss distribution on mode competition is analyzed. Numerical examples are given for a $\mathrm{CO}_{2}$ waveguide laser array. Experimental results of $\mathrm{CO}_{2}$ laser arrays are found to be consistent with the theory, and robust in-phase coupled mode array operation has been achieved.
\end{abstract}

\section{INTRODUCTION}

$\mathbf{M}$ ODE SELECTION has been an important part in the study and application of lasers. Traditionally, the selection of modes is necessary to obtain a single frequency with a single transverse mode, which in single-channel lasers is usually the fundamental Gaussian mode and in phase coupled lasers arrays the in-phase coupled array mode [1]-[3]. Recent progress in active photonic switching and logic devices sees the different modes as representing different states, with the oscillation being switched back and forth between them [4], [5]. These modes can be the longitudinal or transverse modes of the laser cavity, including polarization. Usually, the different modes have different field distributions in the laser cavity. The differences in their volume overlap with the cavity gain and loss distributions play an important role in their competition with each other. An understanding of the difference in the overlap and its effect on mode competition is then fundamental to the proper selection of lasing modes. In this paper, we develop a general analytical method to understand the interaction between the modes and the medium, and its effect on mode competition. We then use these results to explain our experimental demonstration of mode control in $\mathrm{CO}_{2}$ waveguide laser arrays. Conditions on gain and loss distributions for competing modes to oscillate are derived and discussed with special attention given to the in-phase coupled and the out-of-phase coupled modes of an array. Section II considers the spatial variation of the cavity loss and gain, and gain saturation. Differential equations for laser mode development in time are derived. Section III studies the stability of single-mode solutions in a two-mode system. The conditions on gain and loss distributions for competing modes to oscillate are derived. Section IV presents numerical

Manuscript received April 29, 1993. This work was supported in part by grant AFOSR-88-0085 from The Air Force Office of Scientific Research.

Y. Zhang is with Synrad, Inc., Bothell, WA 98011. He was with the California Institute of Technology, Pasadena, CA 91125, when the work in this paper was done.

W. B. Bridges is with the California Institute of Technology, Pasadena, CA 91125

IEEE Log Number 9215247.

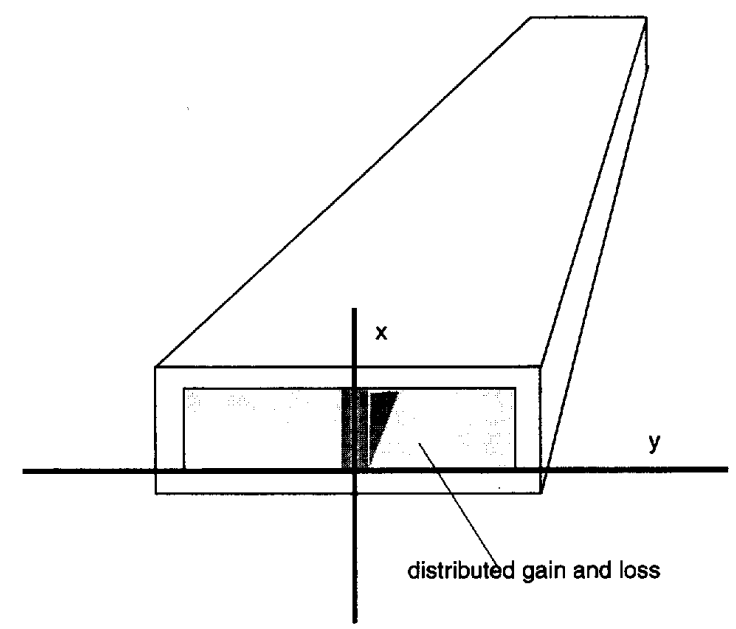

Fig. 1. Schematic showing the general structure of the waveguide array of two channels with distributed gain and loss.

examples of competition between the in-phase and out-ofphase array modes in a dual-channel $\mathrm{CO}_{2}$ laser array. Section $\mathrm{V}$ compares experimental results from two $\mathrm{CO}_{2}$ laser arrays with different structures, one of which is based on the derived conditions to enhance the in-phase coupled mode. A summary is given in Section VI.

\section{MODEL AND THEORY}

We concentrate our attention to the coupled waveguide laser array, the general form of which is shown in Fig. 1. For this model, Maxwell's equations can be written as [6]:

$$
\left.\begin{array}{l}
\nabla \times \boldsymbol{H}=\boldsymbol{J}+\epsilon \frac{\partial \boldsymbol{E}}{\partial t}+\frac{\partial}{\partial t} \boldsymbol{P}_{\text {laser }} \\
\nabla \times \boldsymbol{E}=-\mu \frac{\partial \boldsymbol{H}}{\partial t}
\end{array}\right\}
$$

where the current density $J$ is introduced to account for the loss that is not in resonance with the laser (for example, the mirror transmission loss or the loss introduced by the waveguide boundaries, ) and $\boldsymbol{P}_{\text {laser }}$ is the complex polarization of the medium that resonates with the laser.

Using the normal mode expansion of the resonator field, the total electric and magnetic fields can be written as follows:

$$
\left.\begin{array}{c}
\boldsymbol{E}(\boldsymbol{r}, t)=\sum_{j} e_{j}(t) \phi_{j}(\boldsymbol{r}) \\
\boldsymbol{H}(\boldsymbol{r}, t)=\sum_{j} h_{j}(t) \psi_{j}(\boldsymbol{r})
\end{array}\right\}
$$


where the modal field $\phi_{j}(\boldsymbol{r})$ and $\psi_{j}(\boldsymbol{r})$ are dimensionless and are normalized to the volume of the cavity $V$

$$
\left.\begin{array}{l}
\frac{1}{V} \int_{Y} \phi_{j}(\boldsymbol{r}) \cdot \phi_{k}(\boldsymbol{r}) d v=\delta_{j k} \\
\frac{1}{V} \int_{V} \psi_{j}(\boldsymbol{r}) \cdot \psi_{k}(\boldsymbol{r}) d v=\delta_{j k}
\end{array}\right\}
$$

The loss and the resonant polarization become

$$
\left.\begin{array}{rl}
\boldsymbol{J} & =\sigma(\boldsymbol{r}) \sum_{i} e_{j}(t) \phi_{j}(\boldsymbol{r}) \\
\boldsymbol{P}_{\text {laser }} & =\chi\left(\boldsymbol{r}, \omega_{j}\right) \sum_{j} e_{j}(t) \phi_{j}(\boldsymbol{r})
\end{array}\right\} .
$$

The formal conductivity $\sigma(\boldsymbol{r})$ and the complex dielectric susceptibility $\chi(\boldsymbol{r})$ are functions of space here, while in [6] they are treated as constants. The first of Maxwell's equations

$$
\begin{aligned}
\sum_{j} h_{j}(t) \nabla \times \psi_{j}(\boldsymbol{r})= & \sigma(\boldsymbol{r}) \sum_{j} e_{j}(t) \phi_{j}(\boldsymbol{r}) \\
& +\left[\epsilon+\epsilon_{0} \chi(\boldsymbol{r})\right] \sum_{j} \dot{e}_{j}(t) \phi_{j}(\boldsymbol{r}) .
\end{aligned}
$$

Taking the time derivative of (5), we obtain:

$$
\begin{aligned}
\sum_{j} \dot{h}_{j}(t) \nabla \times \dot{\psi}_{j}(\boldsymbol{r})= & \sigma(\boldsymbol{r}) \sum_{j} \dot{e}_{j}(t) \phi_{j}(\boldsymbol{r}) \\
& +\left[\epsilon+\epsilon_{0} \chi(\boldsymbol{r})\right] \sum_{j} \ddot{e}_{j}(t) \phi_{j}(\boldsymbol{r}) .
\end{aligned}
$$

The normal mode fields satisfy Maxwell's equations for the empty, unperturbed resonator, which is charge free, uniform, passive, and lossless

$$
\left.\begin{array}{l}
\nabla \times \phi_{j}(\boldsymbol{r})=-i \omega_{0 j} \mu \psi_{j}(\boldsymbol{r}) \\
\nabla \times \psi_{j}(\boldsymbol{r})=i \omega_{0 j} \epsilon \phi_{j}(\boldsymbol{r})
\end{array}\right\}
$$

where $\omega_{0 j}$ is the normal frequency for the $j$ th mode in the passive cavity. Substituting the first of (7) in the second of Maxwell's equations, we have

$$
e_{j}(t)=-\frac{i}{\omega_{0 j}} \dot{h}_{j}(t)
$$

Using (7) and (8) in (6)

$$
\begin{aligned}
\sum_{j}-\omega_{0 j}^{2} \epsilon e_{j}(t) \phi_{j}(\boldsymbol{r})= & \sigma(\boldsymbol{r}) \sum_{j} \dot{e}_{j}(t) \phi_{j}(\boldsymbol{r}) \\
& +\left[\epsilon+\epsilon_{0} \chi(\boldsymbol{r})\right] \sum_{j} \ddot{e}_{j}(t) \phi_{j}(\boldsymbol{r})
\end{aligned}
$$

and rearranging the above, we obtain

$$
-\epsilon \sum_{j}\left(\omega_{0 j}^{2} e_{j}+\ddot{e}_{j}\right) \phi_{j}(\boldsymbol{r})=\sum_{j}\left[\sigma(\boldsymbol{r}) \dot{e}_{j}+\epsilon_{0} \chi(\boldsymbol{r}) \ddot{e}_{j}\right] \phi_{j}(\boldsymbol{r})
$$

Multiplying both sides of the above equation with $V^{-1} \phi_{k}(\boldsymbol{r})$ and integrating over the volume $V$, we have

$$
-\epsilon\left(\omega_{0 k}^{2} e_{k}+\ddot{e}_{k}\right)=\sum_{j}\left(\sigma_{j k} \dot{e}_{j}+\epsilon_{0} \chi_{j k} \ddot{e}_{j}\right)
$$

where

$$
\left.\begin{array}{rl}
\sigma_{j k} & \equiv \frac{1}{V} \int_{V} \sigma(\boldsymbol{r}) \phi_{j}(\boldsymbol{r}) \cdot \phi_{k}(\boldsymbol{r}) d v \\
\chi_{j k} & \equiv \frac{1}{V} \int_{V} \chi(\boldsymbol{r}) \phi_{j}(\boldsymbol{r}) \cdot \phi_{k}(\boldsymbol{r}) d v
\end{array}\right\} .
$$

We may write $e_{j}(t)$ as

$$
e_{j}(t) \equiv \frac{1}{2} e_{j 0}(t) e^{i \omega_{j} t}+\text { c.c. }
$$

where $\omega_{j}$ is the laser oscillation frequency, and $e_{j 0}$ is the slowly varying part of the time dependent $e_{j}(t)$. Because we assume $e_{j 0}$ varies slowly, $\left|\ddot{e}_{j 0}\right| \ll \omega_{j}\left|\dot{e}_{j 0}\right|$, and the second derivation of $e_{j}(t)$ can be approximated as

$$
\ddot{e}_{j}(t) \simeq \frac{1}{2}\left[-\omega_{j}^{2} e_{j 0}(t)+i 2 \omega_{j} \dot{e}_{j 0}(t)\right] e^{i \omega_{j} t}+\text { c.c. }
$$

and (11) can be written as

$$
\begin{gathered}
-\epsilon\left[\left(\omega_{0 j}^{2}-\omega_{j}^{2}\right) e_{j 0}+i 2 \omega_{j} \dot{e}_{j 0}\right]=\sum_{k}\left[\left(i \omega_{k} e_{k 0}+\dot{e}_{k 0}\right) \sigma_{k j}\right. \\
\left.+\left(-\omega_{k}^{2} e_{k 0}+i 2 \omega_{k} \dot{e}_{k 0}\right) \epsilon_{0} \chi_{k j}\right] .
\end{gathered}
$$

We see from this equation that the spatial variation of the loss and gain causes a direct coupling between different modes. This is distinct from the kind of coupling that is the main object of this paper, in which the interaction is via the crosssaturation of one laser mode by another. Because laser gain and loss can never be truly uniform in space, direct coupling will, in general, be present and must be given due consideration. Only under the conditions that the orthogonality between the modes is not violated by the presence of $\chi$ and $\sigma(12)$, so that the following inequalities are true, can the direct coupling be omitted:

$$
\left.\begin{array}{c}
\left|\int_{V} \phi_{k}(\boldsymbol{r}) \cdot \phi_{j}(\boldsymbol{r}) \chi\left(\boldsymbol{r}, \omega_{j}\right) d v\right| \\
\ll\left|\int_{V} \phi_{j}(\boldsymbol{r}) \cdot \phi_{j}(\boldsymbol{r}) \chi\left(\boldsymbol{r}, \omega_{j}\right) d v\right| \\
\left|\int_{V} \phi_{k}(\boldsymbol{r}) \cdot \phi_{j}(\boldsymbol{r}) \sigma(\boldsymbol{r}) d v\right| \\
\ll\left|\int_{V} \phi_{j}(\boldsymbol{r}) \cdot \phi_{j}(\boldsymbol{r}) \sigma(\boldsymbol{r}) d v\right|
\end{array}\right\} k j .
$$

This is particularly valid in the cases we are treating here, where $\sigma(\boldsymbol{r})$ and $\chi(\boldsymbol{r})$ are nearly symmetrical functions, and the $k$ th and the $j$ th modes are of the opposite spatial symmetry. If $\sigma(\boldsymbol{r})$ and $\chi(\boldsymbol{r})$ are exactly symmetrical, then the left-hand side of (16) becomes exactly zero. With this approximation, (15) becomes

$$
\begin{aligned}
& {\left[i 2 \omega_{j}\left(1+\frac{\chi_{j}}{n^{2}}\right)+\frac{\sigma_{j}}{\epsilon}\right] \dot{e}_{j 0}} \\
& =\left(-\omega_{0 j}^{2}+\omega_{j}^{2}+\frac{\chi_{j}}{n^{2}} \omega_{j}^{2}-i \frac{\omega_{j} \sigma_{j}}{\epsilon}\right) e_{j 0}
\end{aligned}
$$

where $n$ is the index of refraction of the medium in the absence of gain, and $\sigma_{j}$ and $\chi_{j}$ are shorthand for $\sigma_{i j}$ and $\chi_{j j}$, respectively. The modal susceptibility $\chi_{j}$ can be further written in its real and imaginary parts:

$$
\begin{aligned}
\chi_{j} & \equiv \frac{1}{V} \int_{V} \phi_{j}(\boldsymbol{r}) \cdot \phi_{j}(\boldsymbol{r}) \chi\left(\boldsymbol{r}, \omega_{j}\right) d v \\
& =\frac{1}{V} \int_{V} \phi_{j}(\boldsymbol{r}) \cdot \phi_{j}(\boldsymbol{r})\left(\chi^{\prime}-i \chi\right) d v .
\end{aligned}
$$


When we require $\dot{e}_{j 0}=0$ in (17), we obtain the steady-state solution, which turns into two conditions for steady-state laser oscillation, the first for phase and the second for amplitude

$$
\omega_{j}=\frac{\omega_{0 j}}{\sqrt{1+\frac{\operatorname{Re}\left(\chi_{j}\right)}{n^{2}}}}, \quad \operatorname{Im}\left(\chi_{j}\right)=\frac{\sigma_{j}}{\epsilon_{0} \omega_{j}} .
$$

To simplify the problem further, we consider only homogeneously-broadened media, since as exemplified in Section IV, we will treat $\mathrm{CO}_{2}$ lasers, which are essentially homogeneously broadened. Thus the imaginary part of $\chi_{j}$ can be written as

$$
\begin{aligned}
\operatorname{Im}\left(\chi_{j}\right) & =-\frac{1}{V} \int_{V}\left|\phi_{j}(\boldsymbol{r})\right|^{2} \chi(\boldsymbol{r}) d v \\
& =\frac{1}{V} \frac{n c}{\omega_{j}} \int_{V} \frac{\gamma_{0}(\boldsymbol{r})\left|\phi_{j}(\boldsymbol{r})\right|^{2} d v}{1+\frac{|\boldsymbol{E}(\boldsymbol{r}, t)|^{2}}{2 \eta I_{s}}}
\end{aligned}
$$

where the total electric field $\boldsymbol{E}(\boldsymbol{r}, t)$ is given by (2), and the wave impedance of space filled with dielectric material of permittivity $\epsilon=\epsilon_{r} \epsilon_{0}$ is defined as

$$
\eta \equiv \sqrt{\frac{\mu_{0}}{\epsilon_{r} \epsilon_{0}}} .
$$

The relationship between $\chi(r)$ and the medium gain, the definitions of small signal gain $\gamma_{0}\left[\mathrm{~m}^{-1}\right]$ and homogeneous saturation intensity $I_{s}\left[\mathrm{~W} / \mathrm{m}^{2}\right]$, and their relations to the laser atomic parameters are given in [6], and are cited in the Appendix. Solving (17) for $\dot{e}_{j}$ and substituting laser frequency $\omega_{j}$ as given in the first of (19), we

$$
\dot{e}_{j 0}=\frac{\left(\frac{\operatorname{Im}\left(\chi_{j}\right)}{n^{2}} \omega_{j}^{2}-i \frac{\omega_{j} \sigma_{j}}{\epsilon}\right)}{i 2 \omega_{j}\left(1+\frac{\chi_{j}}{n^{2}}\right)+\frac{\sigma_{j}}{\epsilon}} e_{j 0} .
$$

For most gas laser media, it is true that $|\chi| \ll 1$, thus $\left(\left|\chi_{j}\right| / n^{2}\right) \ll 1$. If the cavity loss is also small, i.e., $\left(\sigma_{j} / \epsilon\right) \ll$ $2 \omega_{j}$, the denominator above is effectively $i 2 \omega_{j}$ and (22) can be further simplified. Using these simplifications and expanding the electric field using (2) in (20), finally (22) becomes

$$
\begin{aligned}
\dot{e}_{j 0} & =\frac{1}{2}\left[\frac{\operatorname{Im}\left(\chi_{j}\right)}{n^{2}} \omega_{j}-\frac{\sigma_{j}}{\epsilon}\right] e_{j 0} \\
& =\left[\begin{array}{c}
\frac{c}{2 n V} \int_{V} \frac{\gamma_{0}(\boldsymbol{r})\left|\phi_{j}(\boldsymbol{r})\right|^{2} d v}{\frac{1}{2 \eta} \sum_{l} e_{j 0} e_{j 0}^{*} \phi_{l}(\boldsymbol{r}) \cdot \phi_{l}(\boldsymbol{r})}-\frac{\sigma_{j}}{2 \epsilon} \\
1+\frac{I_{s}}{I_{j 0}}
\end{array}\right]
\end{aligned}
$$

It is seen clearly in (23) that the coupling among the modes now exists in the form of cross-saturation through the $e_{j 0} e_{j 0}^{*}$ terms. This is the basis of mode competition. In expressing (22) in the form of (23), some assumptions about the population inversion were made, which are detailed in the derivation of the population inversion in the Appendix.

Equation (23) is a differential equation for laser field coefficients, each including phase and amplitude. Since in analyzing mode competition cases, we are only concerned with the change in the mode intensity, which is a measurable quantity, we will derive equations for intensities from (23). This eliminates information on the phase of each mode, which is of no interest here, and simplifies the treatment further. The intensity coefficient for the $j$ th mode can be defined as:

$$
b_{j} \equiv \frac{e_{j 0} e_{j 0}^{*}}{2 \eta} .
$$

It represents the power density $\left[\mathrm{W} / \mathrm{m}^{2}\right]$ for the $j$ th mode, and its differential equation is

$$
\left.\begin{array}{rl}
\dot{b}_{j} & =\frac{d}{d t} \frac{e_{j 0} e_{j 0}^{*}}{2 \eta}=\frac{1}{2 \eta}\left(\dot{e}_{j 0} e_{j 0}^{*}+e_{j 0} \dot{e}_{j 0}^{*}\right) \\
& =2\left[\frac{c}{2 n V} \int_{V} \frac{\gamma_{0}(\boldsymbol{r})\left|\phi_{j}(\boldsymbol{r})\right|^{2} d v}{\sum_{l} b_{j} \phi_{l}(\boldsymbol{r}) \cdot \phi_{l}(\boldsymbol{r})}-L_{j}\right] b_{j} . \\
1+\frac{I_{s}}{l}
\end{array}\right]
$$

In deriving (25), we used the fact that the square bracket term is real. We also introduced a modal loss coefficient

$$
L_{j} \equiv \frac{\sigma_{j}}{2 \epsilon} \text {. }
$$

\section{Mode Stability ANalysis}

We may now use the results of the previous section to investigate the stability of a given mode when it is possible for a second mode, i.e., one with a different transverse field distribution $\phi_{j}(\boldsymbol{r})$, to oscillate. We make the following assumptions.

1) There are only two modes that are sufficiently near the threshold to be appreciably excited. The total field is then:

$$
\boldsymbol{E}(\boldsymbol{r}, t)=e_{10}(t) e^{i \omega_{1} t} \phi_{1}(\boldsymbol{r})+e_{20}(t) e^{i \omega_{2} t} \phi_{2}(\boldsymbol{r}) .
$$

2) The $z$-dependence of the modal fields is in the form of a standing wave and is the same for all modes considered, since they have the same longitudinal mode number. Thus, the spatial hole burning resulted from this fine standing wave pattern in the $z$-direction does not favor one mode over the other and can be ignored.

3) The gain does not depend on $z$. Thus the integral in (23) is only affected by the field variations in the transverse direction $(x, y)$.

4) The loss $\sigma(\boldsymbol{r})$ is divided into a spatially varying part and a constant part

$$
\sigma(\boldsymbol{r})=\sigma^{\prime}(\boldsymbol{r})+\sigma_{0}
$$

With these assumptions, the effects of the localized loss and the distributed loss on laser mode competition can be separated. The modal loss is then

$$
L_{j}=\frac{1}{2 \epsilon V} \int_{V} \sigma^{\prime}(\boldsymbol{r})\left|\phi_{j}(\boldsymbol{r})\right|^{2} d v+\frac{\sigma_{0}}{2 \epsilon} \equiv L_{j}^{\prime}+L_{0} .
$$

To obtain steady-state solutions for (25), we require $\dot{b}_{j}=0$ for $j=1,2$. Then on the right-hand side either the term in 
square brackets is zero or $b_{j}=0$. All possibilities considered, we will have three nontrivial steady-state solutions as follows:

$$
\left(b_{1}, b_{2}\right)=(f, 0), \quad(0, g), \quad \text { or }\left(p_{1}, p_{2}\right) .
$$

We need to analyze the stability of these solutions and find the conditions for them to be stable and unstable. We will only treat the single mode solutions $(f, 0)$ and $(0, g)$ in this paper since two-mode oscillation results in more mathematical complexity and will be a subject of future study. In our experiment, we could obtain oscillations corresponding to $(f, 0)$ and $(0, g)$, as discussed in Section V.

First, consider the stability of the state $(f, 0)$. Under perturbation $\left(\delta_{1}, \delta_{2}\right)$, this state becomes $\left(f+\delta_{1}, \delta_{2}\right)$. We use (25) to find equations for the perturbation $\delta_{1}$ and $\delta_{2}$

$\left.\begin{array}{l}\dot{\delta}_{1}=\left[\frac{c}{2 n S} \int_{S} \frac{\gamma_{0}(x, y)\left|\phi_{1}(x, y)\right|^{2} d x d y}{1+\frac{\left(f+\delta_{1}\right)\left|\phi_{1}\right|^{2}+\delta_{2}\left|\phi_{2}\right|^{2}}{I_{s}}}-L_{1}\right]\left(f+\delta_{1}\right) \\ \dot{\delta}_{2}=\left[\frac{c}{2 n S} \int_{S} \frac{\gamma_{0}(x, y)\left|\phi_{2}(x, y)\right|^{2} d x d y}{1+\frac{\left(f+\delta_{1}\right)\left|\phi_{1}\right|^{2}+\delta_{2}\left|\phi_{2}\right|^{2}}{I_{s}}}-L_{2}\right.\end{array}\right\}$.

Here $\phi_{1}$ and $\phi_{2}$ are functions of $(x, y)$, and $S$ is the total crosssection area of the laser cavity. Because the perturbations are very small, i.e., $|f| \gg\left|\delta_{1}\right|,\left|\delta_{2}\right|$, we can ignore all the second order $\delta$ terms in (30)

$$
\begin{aligned}
\dot{\delta}_{1} \simeq\left\{\frac{c}{2 n S} \int_{S} \frac{\gamma_{0}(x, y)\left|\phi_{1}(x, y)\right|^{2}}{1+\frac{f\left|\phi_{1}(x, y)\right|^{2}}{I_{s}}}\right. \\
\left.\cdot\left[1-2 f \frac{\delta_{1}\left|\phi_{1}(x, y)\right|^{2}}{I_{s}\left(1+\frac{f\left|\phi_{1}(x, y)\right|^{2}}{I_{s}}\right)}\right] d x d y-L_{1}\right\}\left(f+\delta_{1}\right) .
\end{aligned}
$$

The fact that $(f, 0)$ is a steady-state solution implies that the saturated gain equals the total loss

$$
\frac{c}{2 n S} \int_{S} \frac{\gamma_{0}(x, y)\left|\phi_{1}(x, y)\right|^{2} d x d y}{1+\frac{f\left|\phi_{1}(x, y)\right|^{2}}{I_{s}}}-L_{1}=0 .
$$

So the equations in (30) evolve into the following form:

$\left.\begin{array}{l}\dot{\delta}_{1} \simeq \text { cf } \\ -\frac{c f}{n I_{s} S} \int_{S} \gamma_{0}(x, y)\left|\phi_{1}(x, y)\right|^{2} \frac{\delta_{1}\left|\phi_{1}(x, y)\right|^{2}}{\left(1+\frac{f\left|\phi_{1}(x, y)\right|^{2}}{I_{s}}\right)^{2}} d x d y \\ \dot{\delta}_{2} \simeq\left[\frac{c}{2 n S} \int_{S} \frac{\gamma_{0}(x, y)\left|\phi_{2}(x, y)\right|^{2} d x d y}{1+\frac{f\left|\phi_{1}(x, y)\right|^{2}}{I_{s}}}-L_{2}\right] \delta_{2}\end{array}\right\}$.

We can write (33) in a matrix form by defining a vector $\delta$ :

$$
\delta \equiv\left(\begin{array}{l}
\delta_{1} \\
\delta_{2}
\end{array}\right)
$$

The time derivative of $\delta$ is

$$
\dot{\delta} \equiv\left(\begin{array}{c}
\dot{\delta}_{1} \\
\dot{\delta}_{2}
\end{array}\right)=\left(\begin{array}{ll}
A & B \\
C & D
\end{array}\right)\left(\begin{array}{l}
\delta_{1} \\
\delta_{2}
\end{array}\right)
$$

We see from (31) that the matrix elements $B$ and $C$ are zero. Thus, the eigenvalues for the operating matrix are $A$ and $D$. If both of them are negative, then the vector $\delta$ will not grow, and the steady-state $(f, 0)$ is stable; if either $A$ or $D$ is positive, $(f, 0)$ will be unstable. We also see from (33)

$$
\left.\begin{array}{l}
A=-\frac{c f}{n I_{s} S} \int_{S} \frac{\gamma_{0}(x, y)\left|\phi_{1}(x, y)\right|^{4} d x d y}{\left(1+\frac{f\left|\phi_{1}(x, y)\right|^{2}}{I_{s}}\right)^{2}}<0 \\
D=\frac{c}{2 n S} \int_{S} \frac{\gamma_{0}(x, y)\left|\phi_{2}(x, y)\right|^{2} d x d y}{1+\frac{f\left|\phi_{1}(x, y)\right|^{2}}{I_{s}}}-L_{2}
\end{array}\right\} .
$$

The sign of $D$ is not known until $\gamma_{0}(x, y), \phi_{1}(x, y), \phi_{2}(x, y)$, and $\sigma^{\prime}(x, y)$ are specified. We see that because $A$ is negative, the sign of $D$ determines the stability of the solution $(f, 0)$ : if it is positive(negative), this state is unstable(stable).

Similar arguments obviously apply to the other singlemode steady-state $(0, g)$, which becomes $\left(\delta_{1}, g+\delta_{2}\right)$ with perturbation. The equations are

$$
\begin{aligned}
\dot{\delta}_{1}= & {\left[\frac{c}{2 n S} \int_{S} \frac{\gamma_{0}(x, y)\left|\phi_{1}(x, y)\right|^{2} d x d y}{1+\frac{g\left|\phi_{2}(x, y)\right|^{2}}{I_{s}}}-L_{1}\right] \delta_{1} } \\
\dot{\delta}_{2}= & -\frac{c g}{n I_{s} S} \int_{S} \gamma_{0}(x, y)\left|\phi_{2}(x, y)\right|^{2} \\
& \cdot \frac{\delta_{2}\left|\phi_{2}(x, y)\right|^{2}}{\left(1+\frac{g\left|\phi_{2}(x, y)\right|^{2}}{I_{s}}\right)^{2}} d x d y
\end{aligned}
$$

We define the self-saturated modal gain $S_{k}$ and crosssaturated modal gain $C_{k j}$, both in unit of $s^{-1}$, as follows:

$$
\left.\begin{array}{c}
S_{k}=\frac{c}{2 n S} \int_{S} \frac{\gamma_{0}(x, y)\left|\phi_{k}(x, y)\right|^{2} d x d y}{1+\frac{\alpha_{k}\left|\phi_{k}(x, y)\right|^{2}}{I_{s}}} \\
C_{k j}=\frac{c}{2 n S} \int_{S} \frac{\gamma_{0}(x, y)\left|\phi_{j}(x, y)\right|^{2} d x d y}{1+\frac{\alpha_{k}\left|\phi_{k}(x, y)\right|^{2}}{I_{s}}}
\end{array}\right\} \begin{aligned}
& \alpha_{1} \equiv f ; \quad \alpha_{2} \equiv g, \\
& k, j=1,2 ; k \neq j,
\end{aligned}
$$

definitions similar to those used by Tang et al. [4]. Then we may summarize the conditions for the existence and stability of the $j$ th and $k$ th modes: For the $j$ th mode to exist

$$
S_{j}-L_{j}^{\prime}-L_{0}=0 \text {. }
$$

For the $k$ th mode to exist

$$
S_{k}-L_{k}^{\prime}-L_{0}=0 \text {. }
$$

The stability of the $j$ th mode is determined by

$$
C_{j k}-L_{k}^{\prime}-L_{0}\left\{\begin{array}{cl}
<0 & \text { stable } \\
>0 & \text { unstable. }
\end{array}\right.
$$

The stability of the $k$ th mode is determined by

$$
C_{k j}-L_{j}^{\prime}-L_{0} \begin{cases}<0 & \text { stable } \\ >0 & \text { unstable. }\end{cases}
$$




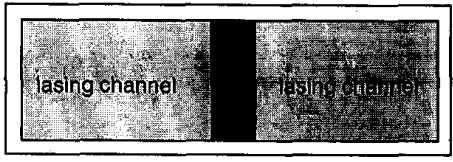

(a)

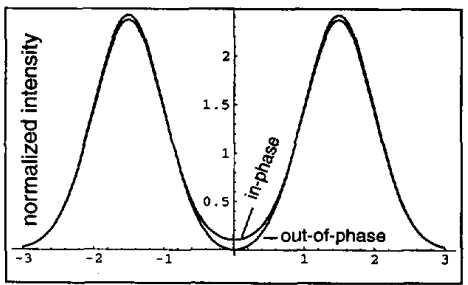

(b)

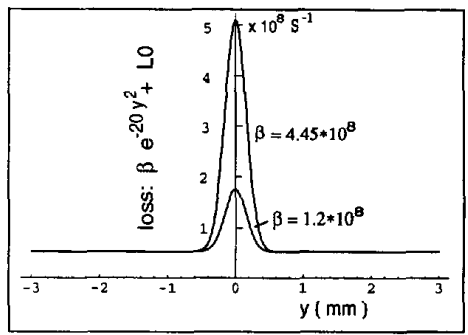

(c)

Fig. 2. (a) Schematic cross section of the waveguide array; (b) the assumed intensity distributions for the in-phase and out-of-phase coupled mode; (c) the assumed loss function for two different values of $\beta$.

Thus, for the laser to have a single $j$ th mode, $(38 \mathrm{c})$ must be $<0$ and (38d) must be $>0$, and vice versa for a single $k$ th mode to exist.

\section{NUMERICAL EXAMPLES}

In this section, we consider numerical examples using parameters typical of $\mathrm{CO}_{2}$ waveguide lasers. The model is an array of two channels coupled by a lossy region, as shown in Fig. 2(a). The competition between the in-phase and outof-phase coupled modes is considered, based on the stability of the $(f, 0)$ and $(0, g)$ states.

The fields are assumed to vary only in $y$-direction; they are assumed to be uniform in $x$-direction (Fig. 2(b)). The small signal gain is assumed to be a constant within the whole array. The loss distribution, concentrated in the coupling region, is also assumed to vary only in $y$, and its amplitude is varied in order to affect the mode competition (Fig. 2(c)). The $x$ dimension is taken as $2 \mathrm{~mm}$, and the $y$-dimension is $6 \mathrm{~mm}$. The small-signal gain $\gamma_{0}$ and loss coefficient that is equivalent to $2 L_{0} / c$ are estimated for a waveguide $\mathrm{CO}_{2}$ laser array to be $0.0012 \mathrm{~mm}^{-1}$ and $0.00034 \mathrm{~mm}^{-1}$, respectively. The saturation intensity is taken from experimental data [7] to be $I_{s}=80$ [watt $\mathrm{mm}^{-2}$ ] at pressures of around 110 torr. The following two functions are used to represent the general forms of the
TABLE I

SUMMARY OF STABILITY FOR THE Two SUPERMODES

\begin{tabular}{ll}
\hline$\beta=1.2 \cdot 10^{8} \mathrm{~s}^{-1}$ & $\beta=4.45 \cdot 10^{8} \mathrm{~s}^{-1}$ \\
\hline stable in-phase mode & stable out-of-phase mode \\
$S_{1}-L_{1}=0 \mathrm{~s}^{-1}$ & $S_{2}-L_{2}=0 \mathrm{~s}^{-1}$ \\
$C_{12}-L_{2}=-7.6 \cdot 10^{5}<0 \mathrm{~s}^{-1}$ & $C_{21}-L_{1}=-1.1 \cdot 10^{6}<0 \mathrm{~s}^{-1}$ \\
$f=144 \mathrm{~W} / \mathrm{mm}^{2}$ & $g=137.6 \mathrm{~W} / \mathrm{mm}^{2}$ \\
unstable out-of-phase mode & unstable in-phase mode \\
$S_{2}-L_{2}=0 \mathrm{~s}^{-1}$ & $S_{1}-L_{1}=0 \mathrm{~s}^{-1}$ \\
$C_{21}-L_{1}=1.04 \cdot 10^{5}>0 \mathrm{~s}^{-1}$ & $C_{12}-L_{2}=1.4 \cdot 10^{6}>0 \mathrm{~s}^{-1}$ \\
$g=140 \mathrm{~W} / \mathrm{mm}^{2}$ & $f=133 \mathrm{~W} / \mathrm{mm}^{2}$ \\
\hline
\end{tabular}

in-phase and out-of-phase coupled modes:

$$
\left.\begin{array}{l}
\phi_{1}(y)=c_{1}\left[\exp \left(-(y-1.5)^{2}\right)+\exp \left(-(y+1.5)^{2}\right)\right] \\
\phi_{2}(y)=c_{2}\left[\exp \left(-(y-1.5)^{2}\right)-\exp \left(-(y+1.5)^{2}\right)\right]
\end{array}\right\}
$$

where $c_{1}=1.540, c_{2}=1.557$ are normalized constants. These modes are plotted in Fig. 2(b). Although they are not the real solutions, these two functional forms resemble the general shape of the in-phase and the out-of-phase coupled modes, and the differences between the two modes. When they are substituted into (16), the left-hand side becomes zero. Thus, the omission of the direct coupling terms is justified.

The spatially varying loss is located at the center of the array where the two channels meet, and is assumed to have the following form:

$$
\frac{\sigma^{\prime}(y)}{2 \epsilon_{0}}=\beta \exp \left(-20 y^{2}\right)
$$

where $\beta$ is a parameter with a unit of $\mathrm{s}^{-1}$. We calculated the stability of the two array modes for two different values of $\beta, 1.2 \cdot 10^{8}$, and $4.45 \cdot 10^{8}$. The total loss function for these two cases are plotted in Fig. 2(c). The results of the calculation are given in Table I.

The result clearly indicates that for the smaller loss $(\beta=$ $1.2 \cdot 10^{8}$ ) in the center, the in-phase coupled mode (with subscript 1) is stable and the out-of-phase coupled mode (with subscript 2) is unstable; and for the larger loss $\left(\beta=4: 45 \cdot 10^{8}\right)$ in the center, the out-of-phase coupled mode is stable and the in-phase coupled mode is unstable. Figs. 3 and 4 are plots of the integrands for the two $\beta$ values, showing that the difference in areas comes mainly from the center region where the loss varies.

We can obtain the mode stability readily by a simple graphic method. From (37), we see $S_{1}$ and $C_{12}$ are functions of $f$; and $S_{2}$ and $C_{21}$ are functions of $g$. We plot these functions in Fig. 5. These curves, combined with the horizontal lines representing the total modal losses, show the range of stability for both $(f, 0)$ and $(0, g)$ states. In Fig. 5(a), the intersection A of $L_{1}$ and $S_{1}$ fixes the value of intracavity intensity $f$, which is 144 . This $f$ value, in turn, determines $C_{12}$ at point B. $L_{2}$ is always less than $L_{1}$ because there is less overlap of the outof-phase mode with the central lossy region. Thus if $L_{2}$ lies between $\mathrm{A}$ and $\mathrm{B}$, then $C_{12}<L_{2}$, and according to (38c), the in-phase mode will be stable. This same $L_{2}$ line also crosses the $S_{2}$ curve at point $C$, setting $g=140$ for the out-of-phase 


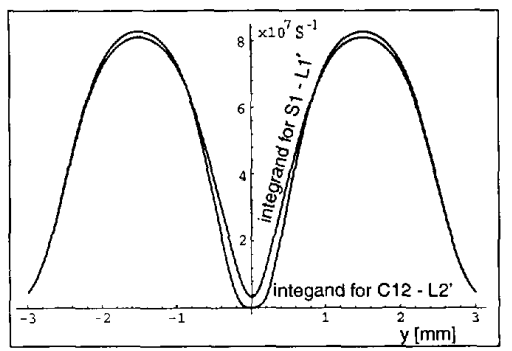

(a)

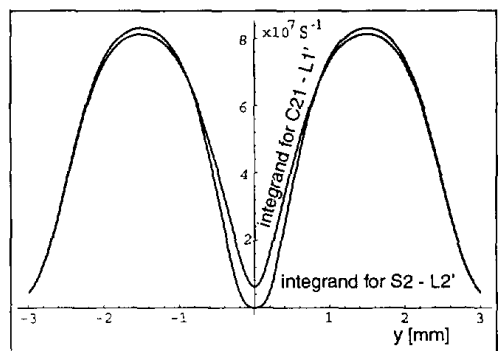

(b)

Fig. 3. Integrand comparison for $\beta=1.2 \cdot 10^{8}$, stable in-phase coupled mode and unstable out-of-phase coupled mode. The larger areas for $S_{1}-L_{1}^{\prime}$ in (a) and $C_{21}-L_{1}^{\prime}$ in (b) are due to contributions from the center region.

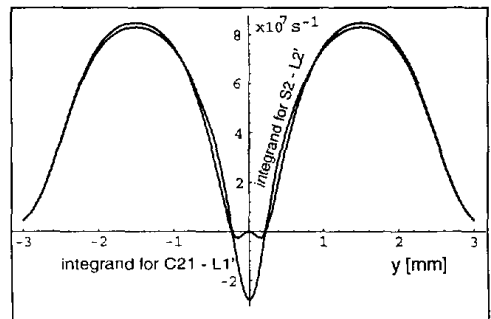

(a)

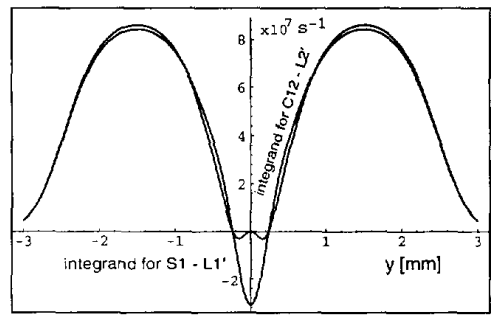

(b)

Fig. 4. Integrand comparison for $\beta=4.45 \cdot 10^{8}$, stable out-of-phase coupled mode and unstable in-phase coupled mode. The larger areas for $S_{2}-L_{2}^{\prime}$ in (a) and $C_{12}-L_{2}^{\prime}$ in (b) are also due to contributions from the center region.

coupled mode. This $g$ value in turn yields $C_{21}$ at point $\mathrm{D}$, which satisfies $C_{21}>L_{1}$, and according to $(38 \mathrm{c}),(0, g)$ state is unstable. Fig. $5(\mathrm{~b})$ shows a different case, where $L_{1}$ and $L_{2}$ are farther apart, corresponding to a larger $\beta$ value. Here the $L_{2}$ line is below point $\mathrm{B}$, and apparently the $C_{12}$ value

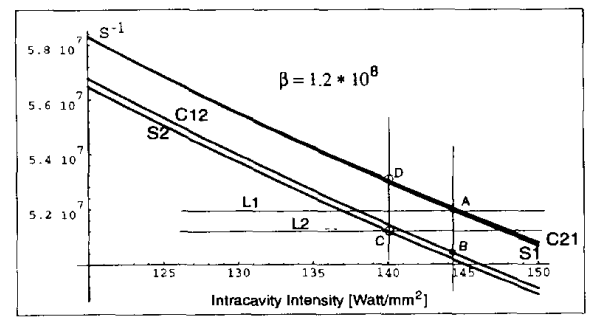

(a)

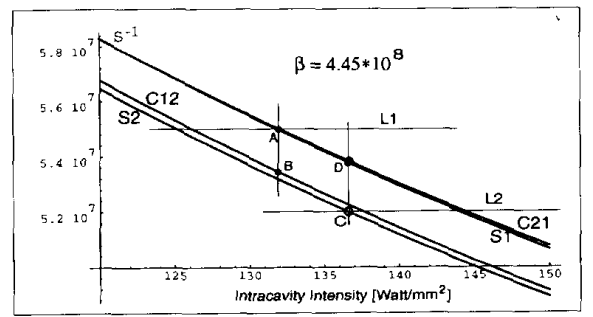

(b)

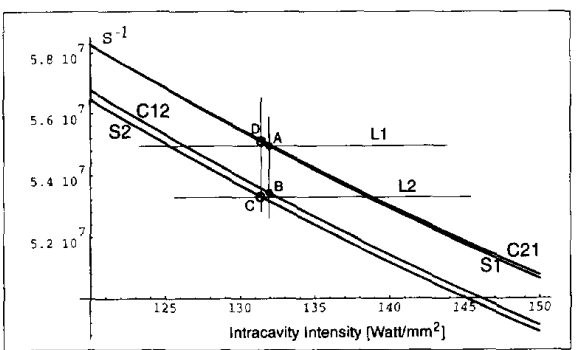

(c)

Fig. 5. The stability of the modes can be derived readily from these diagrams, where $S_{1}, C_{12}, S_{2}$, and $C_{21}$ are plotted as functions of intensity. Intersection A of $S_{1}$ and $L_{1}$ determines the in-phase mode intensity. The position of the $L_{2}$ line lying above or below $B$ determines whether the in-phase mode is stable or not. Intersection $\mathrm{C}$ of $S_{2}$ and $L_{2}$ determines the out-of-phase intensity. The position of the $L_{1}$ line above or below D determines whether the out-of-phase coupled mode is stable or unstable. Thus (a) represents a situation with a stable in-phase mode and an unstable out-phase coupled mode, (b) vice versa and in (c) both modes are unstable.

at $\mathrm{B}$ is larger than $L_{2}$. Thus the in-phase coupled mode is unstable. The same $L_{2}$ line crosses $S_{2}$ at point $\mathrm{C}$, which also determines $g$. The $C_{21}$ value for this $g$ is given by intersection $\mathrm{D}$, lying below the $L_{1}$ line. Thus the out-of-phase coupled mode is stable. The various values at the intersections on Fig. 5(a) and 5(b) are the same as listed in Table I.

This stability diagram reveals yet another possibility, i.e., a situation where neither mode is stable. In Fig. 5(c), the $L_{2}$ line is just slightly bellow point $\mathrm{B}$ so intersection $\mathrm{C}$ is to the left of $\mathrm{B}$, the intersection $\mathrm{D}$ slightly higher than the $L_{1}$ line. Thus, we have $C_{12}>L_{2}$ and $C_{21}>L_{1}$ and both modes are unstable. In our experiments, we have observed the laser switching continuously between the two modes, not stable in either one. This may correspond to the situation described in Fig. 5(c).

We used in the above constant small-signal gain that corresponds to a uniform excitation and the loss function assumed 
in (40) that corresponds to a nonuniform and nonsaturable loss in the laser array. It is also possible, even likely, that the excitation is actually not uniform, eg., there is a smaller gain (or even no gain) in the central coupling region compared to the laser channels. To reflect this situation, we made calculations with a saturable loss in the central region, with a small signal gain

$$
\gamma_{0}(y)=\gamma_{0}^{\prime}-\beta \exp \left(-20 y^{2}\right),
$$

and the localized loss $\sigma^{\prime}(y)$ was taken as zero. The results for two different values of $\beta$ showed the same qualitative trend as for the unsaturated loss case, i.e., for larger (smaller) $\beta$ values within a certain range, the out-of-phase (in-phase) coupled mode is stable and the in-phase (out-of-phase) coupled mode unstable.

Another interesting observation to be made from these numerical calculations is that only about ten percent of the laser volume is involved to achieve the mode switching: For a total intensity distribution of $5 \mathrm{~mm}$ wide $(-2.5$ to 2.5 ), the dimension over which the gain/loss varies is only less than $0.5 \mathrm{~mm}$. In our experiments with $\mathrm{CO}_{2}$ lasers, we have no convenient way of changing the local gain or loss electronically. But if our calculations are applicable to semiconductor lasers, where there are ways of changing the local injection current, it should be possible to change the oscillation mode electronically. The small volume involved would imply a small capacitance and hence a high switching speed. This might be useful in semiconductor optical logic devices.

\section{EXPERIMENTAL RESULTS}

There have been reports of several designs of phase coupled waveguide $\mathrm{CO}_{2}$ laser arrays [3], [8], [9]. The structure in [8] was chosen for experimental investigation because our previous experience with it. It is shown schematically in Fig. 6(a). The individual waveguide laser channels are coupled by optical leakage through the slots in the side-walls separating the channels. The material used for the waveguide channels can be ceramic, as in [8], or metal, as in [10], whre the metal array bed and the metal top electrode are separated by a thin layer of insulating material. The type of structure illustrated in Fig. 6(a) introduces a considerable loss in the coupling region because the wall material is lossy at the laser wavelength. This corresponds to a large $\beta$ in the analysis of Section IV. Consequently, this type of array structure greatly favors the out-of-phase mode rather than the in-phase coupled mode. In a structure that would favor the in-phase coupled mode, there must be only a small optical loss in the coupling region, i.e., a small $\beta$, yet retaining separate channels.

We conceived a new structure that satisfies this requirement. In this type of coupled laser array, as shown in Fig. 6(b), strip waveguides replace the hollow waveguides as the lasing channels. The channels are now coupled by waves leaking across the grooves in the bottom (or top or both) wall. By eliminating the side walls in the separating region, the net loss there is greatly reduced and a small $\beta$ is realized. We tested arrays with two, three and five channels, the strip width being

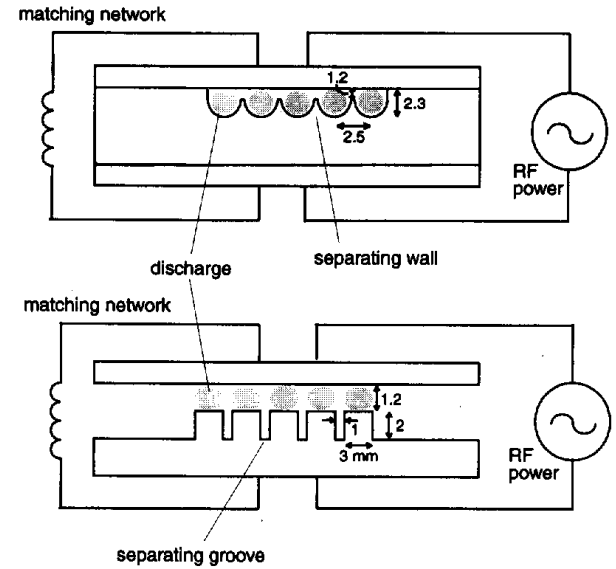

Fig. 6. Comparison of two RF-excited $\mathrm{CO}_{2}$ waveguide laser array structures. (a) A wall-slot-coupled array: the lasing channels are separated by the thin half-height wall. (b) Groove-coupled strip array; the lasing channels are the spaces between the strips and the top plate, and are separated by the narrow grooves in the bottom plate. Typical dimensions are given in millimeters.

3 to $5 \mathrm{~mm}$, the gap between the electrode/waveguide plates 0.8 to $1.2 \mathrm{~mm}$ and grooves $1 \mathrm{~mm}$ to $2 \mathrm{~mm}$ wide and $2 \mathrm{~mm}$ deep.

The mode of an individual element of this new array structure is a waveguide-Gaussian hybrid: the polished strip electrodes provide waveguiding in the narrow dimension; and there are no guiding surfaces in the wide dimension so it is the free space Gaussian mode. Proper selection of the gap size and the width of the strip can yield the lowest order mode in both directions. When two or more of these strips are brought together side-by-side within a proper distance, the tail of the Gaussian distribution of one leaks into the adjacent element(s), causing the phase coupling. At the sharp corners of the strip, the strong RF field bends outward. Thus, the gain medium extends outside the width of the element. As a result, the Gaussian distribution is strong enough outside the elements to produce a stable phase coupling for the array.

One might argue that such a structure is now fundamentally different, since the parallel strips are no longer waveguides, but, in fact, may be "leaky guides," or "antiguides," and no longer amenable to coupled-mode analysis, as in the case of antiguiding semiconductor laser arrays in [1], analyzed with a leaky wave theory by Botez et al. [11]. However, that conclusion is not supported by our experimental observations. If the grooves in Fig. 6(b) are omitted entirely, so that the guiding structure is simply two parallel plates, $20 \mathrm{~mm}$ wide separated by about $2 \mathrm{~mm}$, then what is observed is a high-order cosine-Gaussian mode, as shown in Fig. 7. The experimentally observed intensity patterns are clearly Gaussian in the direction parallel to the plates, with the characteristic nonuniform spacing of the zeros and wider width of the two outer lobes. It resembles the theoretical fourth-order cosine-Gaussian intensity distribution, not the cosine-cosine distribution that would have uniformly spaced zeros and equal width in all high intensity lobes. One can thus conclude that if the width of the slab is reduced to support only the lowest order mode, this mode should also be a cosine-Gaussian mode, not a leaky waveguide mode. 


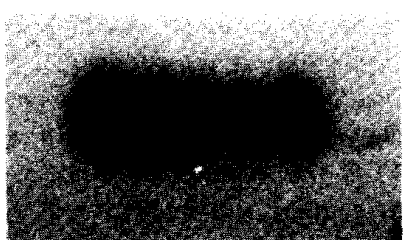

(a)

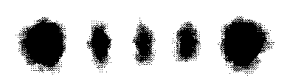

(b)

Fig. 7. (a) The experimental intensity distribution from the wide slab waveguide laser; (b) theoretical fourth-order Hermite-Gaussian distribution in the wide direction and lowest cosine mode in the narrow direction. We can say by comparison that the mode in the slab waveguide laser is the one in (b).

If very thin grooves are cut in one of the wide plates, the high-order cosine-Gaussian mode persists, ignoring the grooves. For example, Fig. 8(a) shows a Gaussian mode with 3 zeros originating from a structure with two $0.5 \mathrm{~mm}$ wide grooves. However, if the two grooves are made slightly wider, eg., to $1 \mathrm{~mm}$, then the near field changes to Fig. $8(\mathrm{~b})$, where the number of zeros is the same as the number of grooves cut. We interpret this as a shift to the lowest order cosine-Gaussian mode oscillating in each strip. The far-field patterns and spectrum analyzer beat spectrum confirm that this is indeed a single mode, with the adjacent maxima all locked in-phase.

In the antiguiding structure of semiconductor laser arrays, the transverse structure has to be at resonance with the laser wavelength in the material in order to arrange the field in all active channels to oscillate at the same phase [11]. In our experiments, however, we used quite different channel widths for different arrays, eg., $3 \mathrm{~mm}, 5 \mathrm{~mm}$, and $6 \mathrm{~mm}$, and we used groove widths from $1.0 \mathrm{~mm}$ to $2.0 \mathrm{~mm}$. They could all be coupled in-phase; no resonance condition behavior depending on the widths of the strips and grooves was observed.

We investigated two-channel arrays of both wall-slot coupled and groove-coupled guides to compare their behavior. The wall-slot-coupled array was made with alumina ceramic channels and an aluminum top plate. This array supported both the in-phase coupled and out-of-phase coupled modes, but the in-phase mode was much less stable than the outof-phase mode. Although either mode would oscillate with a proper cavity alignment, the range of mechanical adjustment for the in-phase mode was much less than that for the out-ofphase mode. When a small piece of lossy material was put on top of the wall, as in Fig. 9(a), blocking the optical path for just a short length, it eliminated the in-phase coupled mode altogether and selected only the out-of-phase coupled mode, no matter how the cavity alignment was adjusted.

A similar two-channel wall-slot coupled array was made of aluminum with a glass top plate. Because of the added fixed loss in the side walls, this laser would oscillate only in the out-of-phase coupled mode for any mirror adjustment.
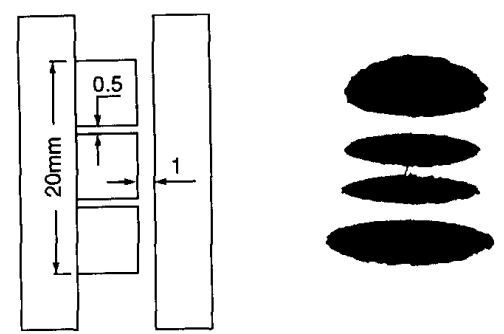

(a)
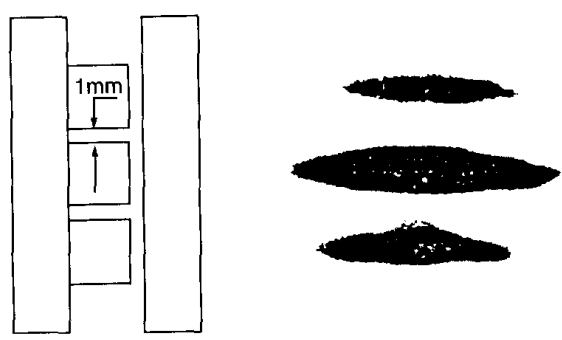

(b)

Fig. 8. The effect of groove width in a three strip array: (a) narrow grooves being ignored by the discharge and the near-field intensity distribution is that for a third order cosine-Gaussian mode. Three nulls do not match two grooves. (b) wider grooves yield a different near-field distribution, lowest order cosine-Gaussian mode in the strips coupled together. Two nulls match the number of grooves.

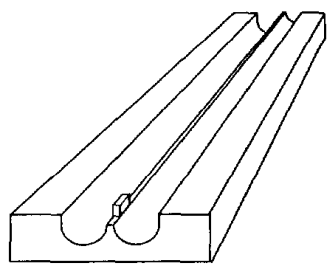

(a)

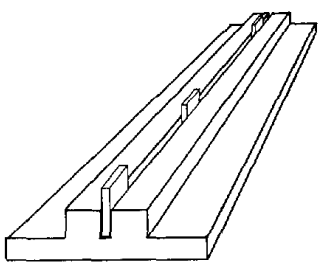

(b)

Fig. 9. Schematics showing location of the lossy material in the center region: (a) of the wall-slot-coupled array, (b) of the groove-coupled strip array.

The relative behavior of the in- and out-of-phase coupled modes in a two-channel groove-coupled array was quite different, however. A two-strip groove-coupled array of the type shown in Fig. 6(b) was made with top and bottom aluminum walls. In this array, the in-phase coupled mode was greatly favored over the out-of-phase mode. Over a wide range of gas pressure, including the optimum pressure for power output, it exhibited only the in-phase coupled mode. In an attempt to test the robustness of this in-phase coupled operation, three lossy 


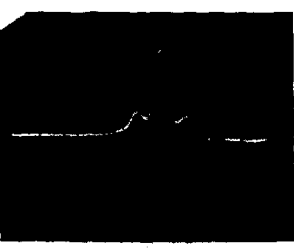

two-channsl array

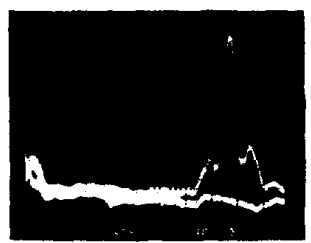

five-channel array

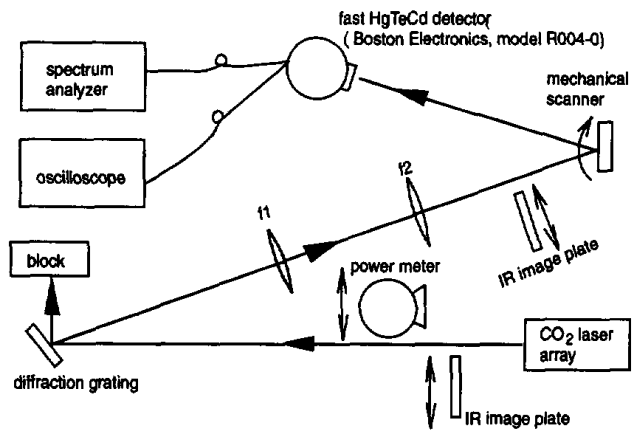

Fig. 10. Far-field intensity scans for the two- and five-channel groove coupled strip array and the measurement setup.

glass pieces were added to the slot as shown in Fig. 9(b). The oscillation remained primarily in the in-phase coupled mode. The out-of-phase coupled mode appeared only transiently at some particular cavity alignments, quickly resorting to the in-phase-coupled mode. This is just the reverse of the wallslot-coupled array behavior.

Fig. 10 shows the far-field scans of the intensity distributions from the two- and five-channel groove-coupled strip waveguide $\mathrm{CO}_{2}$ laser arrays and the measurement setup. A fast photo-detector (Boston Electronics $\mathrm{HgCdTe}$ model R004-0, response time $1 \mathrm{~ns}$ ) and a spectrum analyzer were used to detect beat notes indicating multimode operation or independent channel (uncoupled) oscillation. Both the wallslot-coupled and groove-coupled two-channel lasers were investigated for beat frequencies. The wall-slot-coupled array always exhibited beats indicating multiple transverse modes, in both the predominantly in-phase mode and predominantly out-of-phase mode cases. The beats disappeared when the loss was added to the array center, indicating pure out-of-phase operation. For the groove-coupled array, however, there was a range of cavity adjustment where there were no beat notes on the spectrum analyzer. This, combined with the singlelobe far-field intensity distribution shown in Fig. 10, indicated that we observed true single in-phase mode operation. We can conclude from these results that our new coupled waveguide laser array design is capable of stable single in-phase coupled mode operation, as predicted by the theory in the earlier sections.

\section{SUMMARY}

A theoretical analysis of laser transverse mode competition is developed from the perspective of the spatial overlap of

modes with a transverse gain-loss distribution. The dominant mode of laser oscillation is the mode that is stable under small perturbations. The conditions that this mode must satisfy were derived. Numerical calculations were applied to twochannel coupled $\mathrm{CO}_{2}$ waveguide array lasers, with the result that a change in gain or loss in the small coupling region between the channels of the array was capable of switching the laser oscillation from one mode to the other. This property of the coupled array may be important in understanding active super mode control. Experimental results on wall-slot-coupled and groove-coupled $\mathrm{CO}_{2}$ laser array structures support the theoretical analysis. Robust in-phase coupled mode oscillation was obtained in two- and five-element arrays with the groovecoupled structure.

\section{APPENDIX}

\section{Saturation of Population Inversion by} Multiple Transverse Mode

Multimode laser oscillation has been treated by Sargent, Scully, and Lamb [12], to whom we owe a great deal for their insight. However, their analysis is based on a small-signal approximation, which is not valid in our situation. Thus, we derive a new relation by starting from Yariv's [6] analysis and expand it to multimode cases. We begin with the differential equations for density matrix elements given in Yariv

$$
\begin{aligned}
\frac{d}{d t} \rho_{21} & =-i \omega_{0} \rho_{21}+i \frac{\mu}{\hbar} E(t)\left(\rho_{11}-\rho_{22}\right)-\frac{\rho_{21}}{T_{2}} \\
\frac{d}{d t}\left(\rho_{11}-\rho_{22}\right)= & i 2 \frac{\mu}{\hbar} E(t)\left(\rho_{21}-\rho_{21}^{*}\right) \\
& -\frac{\left(\rho_{11}-\rho_{22}\right)-\left(\rho_{11}-\rho_{22}\right)_{0}}{\tau}
\end{aligned}
$$

Assume a single longitudinal mode that can oscillate in any of $N$ transverse modes. The frequency differences among these modes are very small compared to the gain line width. Define the linearly polarized electric field as follows:

$$
E(t)=\frac{1}{2} \sum_{j}^{N} e_{j 0}(t) e^{-i \omega_{j} t} \phi_{j}(\boldsymbol{r})+\text { c.c. }
$$

where $e_{j 0}(t)$ is a slowly varying function of time.

We can write the off-diagonal density matrix element $\rho_{21}$ as follows:

$$
\rho_{21}(t) \equiv \sigma_{21}(t) e^{-i \omega_{a} t}, \quad \omega_{a} \equiv \frac{\sum_{j}^{N} \omega_{j}}{N} .
$$

Thus from the (Ala), we obtain the differential equation for $\sigma_{21}$

$$
\dot{\sigma}_{21}=i\left(\omega_{a}-\omega_{0}\right) \sigma_{21}+i \frac{\mu}{2 \hbar}\left(\rho_{11}-\rho_{22}\right) \sum_{j}^{N} e_{j 0} \phi_{j} e^{i \Delta_{a j} t}-\frac{\sigma_{21}}{T_{2}}
$$


where $\Delta_{a j}$ is the frequency difference: $\Delta_{a j} \equiv \omega_{a}-\omega_{j}$. High-frequency terms in (A4) are ignored because their contributions to the integration average to zero over the time scale for variations in $\sigma_{21}$. Multiply both sides of (A4) by the factor $e^{\left[i\left(\omega_{0}-\omega_{a}\right)+\left(1 / T_{2}\right)\right] t}$ and with some manipulation, (A4) becomes

$$
\begin{aligned}
\frac{d}{d t}\left(\sigma_{21} e^{\left[i\left(\omega_{0}-\omega_{a}\right)+\left(1 / T_{2}\right)\right] t}\right)= & i \frac{\mu}{2 \hbar}\left(\rho_{11}-\rho_{22}\right) \sum_{j}^{N} \\
& \cdot e_{j 0} \phi_{j} e^{\left[i\left(\omega_{0}-\omega_{j}\right)+\left(1 / T_{2}\right)\right] t}
\end{aligned}
$$

Assuming the rate of change of $\left(\rho_{11}-\rho_{22}\right)$ and $e_{j 0}$ are much slower than $1 / T_{2}$, we can pull them out of the integration. Then we obtain by integrating (A5)

$$
\sigma_{21}=i \frac{\mu}{2 \hbar}\left(\rho_{11}-\rho_{22}\right) \sum_{j}^{N} e_{j 0} \phi_{j} e^{i \Delta_{a j} t} D\left(T_{2}, \omega_{0}-\omega_{j}\right)
$$

where $D\left(T_{2}, \omega_{0}-\omega_{j}\right)$ is defined as

$$
D\left(T_{2}, \omega_{0}-\omega_{j}\right) \equiv \frac{1}{\frac{1}{T_{2}}+i\left(\omega_{0}-\omega_{j}\right)}
$$

The term $\rho_{21}-\rho_{21}^{*}$ in (A1b) becomes

$$
\begin{aligned}
& \rho_{21}-\rho_{21}^{*}=\sigma_{21}(t) e^{-i \omega_{a} t}-\sigma_{21}^{*}(t) e^{i \omega_{a} t} \\
= & i \frac{\mu}{2 \hbar}\left(\rho_{11}-\rho_{22}\right)\left[\sum_{j}^{N} e_{0} \phi_{j} e^{i \omega_{j} t} D\left(T_{2}, \omega_{0}-\omega_{j}\right)+\text { c.c. }\right] .
\end{aligned}
$$

Thus, we have

$$
\begin{aligned}
\frac{d}{d t}\left(\rho_{11}\right. & \left.-\rho_{22}\right) \\
= & i \frac{\mu}{\hbar} E(t)\left(\rho_{21}-\rho_{21}^{*}\right)-\frac{\left(\rho_{11}-\rho_{22}\right)-\left(\rho_{11}-\rho_{22}\right)_{0}}{\tau} \\
= & -\frac{1}{2}\left(\frac{\mu}{\hbar}\right)^{2}\left(\rho_{11}-\rho_{22}\right)\left[\sum_{j}^{N} e_{j 0} e^{-i \omega_{j} t} \phi_{j}(\boldsymbol{r})+\text { c.c. }\right] \\
& \times\left[\sum_{j}^{N} e_{j 0} \phi_{j}(\boldsymbol{r}) e^{i \omega_{j} t} D\left(T_{2}, \omega_{0}-\omega_{j}\right)+\text { c.c. }\right] \\
& -\frac{\left(\rho_{11}-\rho_{22}\right)-\left(\rho_{11}-\rho_{22}\right)_{0}}{\tau} .
\end{aligned}
$$

If the frequency spread of the transverse modes is small compared with the line width, we can approximate $D\left(T_{2}, \omega_{0}-\right.$ $\left.\omega_{j}\right)$ with $D\left(T_{2}, \omega_{0}-\omega_{a}\right)$. And if the rate of change of $\rho_{11}-\rho_{22}$ is much slower than $e^{i\left(\omega_{j}-\omega_{k}\right) t}$ for any $k \neq j$, we can keep only the terms without exponential time dependence on the right side of (A9)

$$
\begin{aligned}
\frac{d}{d t}\left(\rho_{11}-\rho_{22}\right)= & -\frac{1}{2}\left(\frac{\mu}{\hbar}\right)^{2}\left(\rho_{11}-\rho_{22}\right) \\
& \cdot\left[D\left(T_{2}, \omega_{0}-\omega_{a}\right)+\text { c.c. }\right] \sum_{j}^{N} e_{j 0} e_{j 0}^{*} \phi_{j}^{2}(\boldsymbol{r}) \\
& -\frac{\left(\rho_{11}-\rho_{22}\right)-\left(\rho_{11}-\rho_{22}\right)_{0}}{\tau} \\
= & -\frac{1}{2}\left(\frac{\mu}{\hbar}\right)^{2}\left(\rho_{11}-\rho_{22}\right) \frac{2 T_{2}}{1+T_{2}^{2}\left(\omega_{0}-\omega_{a}\right)^{2}} \\
& \cdot \sum_{j}^{N} e_{j 0} e_{j 0}^{*} \phi_{j}^{2}(\boldsymbol{r}) \\
& -\frac{\left(\rho_{11}-\rho_{22}\right)-\left(\rho_{11}-\rho_{22}\right)_{0}}{\tau} .
\end{aligned}
$$

We make the standard definitions of the normalized line shape function:

$$
g(\omega) \equiv \frac{2 T_{2}}{1+T_{2}^{2}\left(\omega_{0}-\omega_{a}\right)^{2}}
$$

the saturation intensity:

$$
I_{s} \equiv c n \epsilon_{0} \frac{\hbar^{2}}{\mu^{2} \tau g\left(\omega_{a}\right)}=\frac{1}{\eta} \frac{\hbar^{2}}{\mu^{2} \tau g\left(\omega_{a}\right)} .
$$

and the laser intensity:

$$
I \equiv \frac{1}{2 \eta} \sum_{j}^{N} e_{j 0} e_{j 0}^{*} \phi_{j}^{2}(\boldsymbol{r})=\sum_{j}^{N} I_{j}
$$

Now we can write the steady-state expression for $\left(\rho_{11}-\rho_{22}\right)$ as

$$
\rho_{11}-\rho_{22}=\frac{\left(\rho_{11}-\rho_{22}\right)_{0}}{1+\frac{I}{I_{s}}} .
$$

From [6], the susceptibility satisfies the following:

$$
\chi \propto \rho_{11}-\rho_{22}
$$

and

$$
\chi=-\frac{n^{2} c}{\omega} \gamma
$$

where $\gamma$ is the gain coefficient of lasing medium in units of $\left[\mathrm{m}^{-1}\right]$.

\section{ACKNOWLEDGMENT}

The authors wish to acknowledge the encouragement of Richard Hart of United Technologies Optical Systems in the early stages of the work, and particularly the loan of some ceramic wall-slot-coupled pieces for comparison measurements. They also wish to acknowledge helpful discussions with their colleagues at Caltech, Arthur Sheiman and Finbar Sheehy, as well as the strong technical support in the experimental work by Reynold Johnson. 


\section{REFERENCES}

[1] L. J. Mawst, D. Botez, M. Jansen, T. J. Roth, and J. J. Yang, "Highly coherent in-phase mode operation of 20-element resonant arrays of antiguides," IEEE Photonics Tech. Lett., vol. 2, no. 4, pp. 249-252, Apr. 1990.

[2] Y. Zhang and W. B. Bridges, "Stable in-phase locked arrays of $\mathrm{CO} 2$ waveguide lasers," presented at the Optical Society of America 1991 Annual Meeting, San Jose, CA, Nov. 3-8, 1991, paper THJS.

[3] G. L. Bourdet, G. M. Mullot, and J. Y. Vint, "Linear array of selffocusing $\mathrm{CO}_{2}$ waveguide lasers," IEEE J. Quantum Electron., vol. 26 , no. 4, pp. 701-710, Apr. 1990

[4] C. L. Tang, A. Schremer, and T. Fujita, "Bistability in two-mode semiconductor lasers via gain saturation," Appl. Phys. Lett., vol. 51, no. 18, pp. 1392-1394, Nov. 1, 1987.

[5] D. F. G. Gallagher, "Gain-coupled optical logic in semiconductor lasers," Appl. Optics, vol. 29, no. 29, pp. 4359-4371, Oct. 10, 1990.

[6] A. Yariv, Quantum Electronics, 3rd edn., New York: Wiley, 1989.

[7] R. Abrams and W. B. Bridges, "Characteristics of sealed-off waveguide $\mathrm{CO}_{2}$ lasers," IEEE J. Quantum Electron., vol. QE-9, pp. 940-946, Sept. 1973.

[8] L. A. Newman, R. A. Hart, J. T. Kennedy, A. J. Cantor, A. J. DeMaria and W. B. Bridges, "High power coupled $\mathrm{CO}_{2}$ waveguide laser array," Appl. Physics Lett., vol. 48, no. 25, pp. 1701-1703, June 23, 1986.

[9] D. G. Youmans "Phase locking of adjacent channel leaky waveguide $\mathrm{CO}$ 2 lasers," Appl. Physics Lett., vol. 44, no. 4, pp. 365-367, Feb. 15, 1984

[10] W. B. Bridges and Y. Zhang, "Coupled waveguide gas laser research," Annual Report on Contract AFOSR-88-0085, Feb. 28, 1990.

[11] D. Botez, L. J. Mawst, G. L. Peterson and T. J. Roth, "Phaselocked arrays of antiguides: Modal content and discrimination," IEEE $J$. Quantum Electron., vol. 26, no. 3, pp. 482-495, Mar. 1990.

[12] M. Sargent III, M. O. Scully, and W. E. Lamb, Jr., Laser Physics, Cambridge, MA: Addison-Wesley, 1974.

Yongfang Zhang received the B.S. degree in physics in January 1982 from Northwest University, Xi'an, China; the M.S. degree in optical sciences in December 1984 from Huazhong University of Science and Technology, Wuhan, China; and the M.S. and Ph.D. degrees in electrical engineering from the Califomia Institute of Technology in June 1991 and June 1993 , respectively.

He is currently chief scientist at Synrad, Inc., of Bothell, Washington.
William B. Bridges (S'53-M'61-F'70) was born in Inglewood, CA, in 1934 He received the B.S., M.S., and Ph.D. degrees in electrical engineering from the University of California, Berkeley, in 1956, 1957, and 1962, respectively, and was an associate in electrical engineering from 1957 to 1959 , teaching courses in communication and circuits. His graduate research dealt with noise in microwave tubes and with electron-stream instabilities (which later became the basis of the Vircator). Summer jobs at RCA and Varian provided stimulating experience with microwave radar systems, ammonia beam masers, and the early development of the ion vacuum pump.

He joined the Hughes Research Laboratories in 1960 as a member of the technical staff and was a senior scientist from 1968 to 1977 , with a brief tour as manager of the Laser Department in 1969-70. His research at Hughes involved gas lasers of all types and their application to optical communication, radar, and imaging systems. $\mathrm{He}$ is the discoverer of laser oscillation in noble gas ions and spent several years on the engineering development of practical highpower visible and ultraviolet ion lasers for military applications. He joined the faculty of the California Institute of Technology in 1977 as professor of electrical engineering and applied physics, serving as executive officer for electrical engineering from 1979 to 1981. In 1983, he was appointed Carl F. Braun Professor of Engineering, and conducts research in optical and millimeter-wave devices and their applications. Current studies include the millimeter-wave modulation of light and innovation in gas lasers.

Dr. Bridges is a member of Eta Kappa Nu, Tau Beta Pi, Phi Beta Kappa, and Sigma $\mathrm{Xi}$, receiving Honorable Mention from Eta Kappa Nu as an "Outstanding Young Electrical Engineer" in 1966. He received the Distinguished Teaching Award in 1980 and 1982 from the Associated Students of Caltech, the Arthur L. Schawlow Medal from the Laser Institute of America in 1986, and the IEEE LEOS Quantum Electronics Award in 1988. He is a member of the National Academy of Engineering and the National Academy of Sciences, and a Fellow of the Optical Society of America and the Laser Institute of America. He was a Sherman Fairchild Distinguished Scholar at Caltech in 1974-75, and a visiting professor at Chalmers Technical University, Göteborg, Sweden in 1989. He is coauthor (with C. K. Birdsall) of Electron Dynamics of Diode Regions (New York: Academic Press, 1966). He has served on various committees of both IEEE and OSA, and was formerly associate editor of the IEEE Journal of Quantum Electronics and the Journal of the Optical Society of America. He was the president of the Optical Society of America in 1988, and was a member of the U.S. Air Force Scientific Advisory Board, 1985-89. 